Nouveaux cahiers de la recherche en éducation

\title{
Comparaison des facteurs personnels, scolaires et familiaux associés aux troubles intériorisés à la fin du primaire et au début du secondaire
}

\author{
Diane Marcotte, Marilou Cournoyer, Marie-Ève Gagné et Marianne Bélanger
}

Volume 8, numéro 2, 2005

Groupe de recherche sur les inadaptations sociales de l'enfance

URI : https://id.erudit.org/iderudit/1017529ar

DOI : https://doi.org/10.7202/1017529ar

Aller au sommaire du numéro

Éditeur(s)

Faculté d'éducation, Université de Sherbrooke

ISSN

1911-8805 (numérique)

Découvrir la revue

Citer cet article

Marcotte, D., Cournoyer, M., Gagné, M.-È. \& Bélanger, M. (2005). Comparaison des facteurs personnels, scolaires et familiaux associés aux troubles

intériorisés à la fin du primaire et au début du secondaire. Nouveaux cahiers de la recherche en éducation, 8(2), 57-67. https://doi.org/10.7202/1017529ar
Résumé de l'article

Cet article présente les résultats de deux études qui explorent les caractéristiques personnelles, familiales et scolaires associées aux troubles intériorisés à la fin du primaire et au début du secondaire. Les mesures ont été complétées par des élèves de $6^{\mathrm{e}}$ année du primaire et de $2^{\mathrm{e}}$ secondaire ainsi que par leur enseignant. Les résultats permettent d'associer plusieurs caractéristiques, telles que les distorsions cognitives, une faible cohésion familiale et la présence de conflits familiaux chez les élèves dépressifs et anxieux. D'autres caractéristiques, telles que le soutien des amis et les habiletés sociales, les problèmes d'apprentissage et de comportement, les délits et le rendement scolaire distinguent les jeunes dépressifs ou anxieux des groupes témoins selon l'ordre scolaire. Des pistes d'intervention sont explorées. 


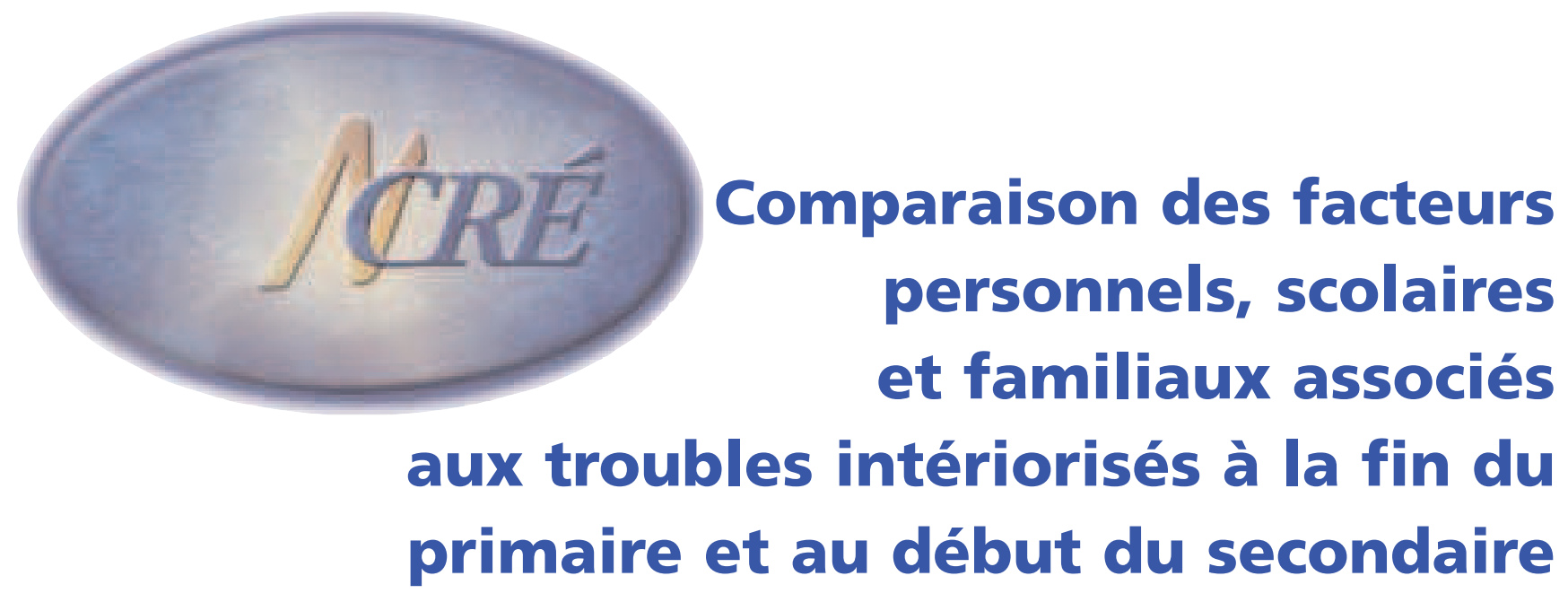

\author{
Diane Marcotte, Marilou Cournoyer, \\ Marie-Ève Gagné et Marianne Bélanger \\ Université du Québec à Montréal
}

Résumé - Cet article présente les résultats de deux études qui explorent les caractéristiques personnelles, familiales et scolaires associées aux troubles intériorisés à la fin du primaire et au début du secondaire. Les mesures ont été complétées par des élèves de $6^{\mathrm{e}}$ année du primaire et de $2^{\mathrm{e}}$ secondaire ainsi que par leur enseignant. Les résultats permettent d'associer plusieurs caractéristiques, telles que les distorsions cognitives, une faible cohésion familiale et la présence de conflits familiaux chez les élèves dépressifs et anxieux. D'autres caractéristiques, telles que le soutien des amis et les habiletés sociales, les problèmes d'apprentissage et de comportement, les délits et le rendement scolaire distinguent les jeunes dépressifs ou anxieux des groupes témoins selon l'ordre scolaire. Des pistes d'intervention sont explorées.

Abstract - This article presents the findings of two studies that explored the personal, family, and academic characteristics associated with internalized disorders at the end of elementary and the start of secondary school. Measures were taken for grade six pupils, second-year secondary pupils, and their teachers. Our findings show associations among several characteristics, such as cognitive distortions, weak family cohesion, and the presence of family conflict, for depressive and anxious pupils. Other characteristics, such as support from friends and social skills, learning and behavioural problems, and criminal acts and academic performance, also distinguish depressive and anxious youths from the control group, depending on the school's organization. The article explores avenues for intervention. 


\section{Problématique}

Aujourd'hui, dans une école secondaire moyenne de 1000 élèves, et ce, à n'importe quel point dans le temps, on peut compter 50 élèves ayant un trouble dépressif majeur, 350 vivant des attaques de panique et jusqu'à dix qui vont commettre une tentative de suicide dans l'année (Atkinson et Hornby, 2002). Les troubles dépressifs et anxieux sont d'ailleurs parmi les problèmes les plus fréquemment traités par les psychologues scolaires. Cet article s'intéresse aux facteurs associés à ces troubles à la fin du primaire et au début du secondaire.

\subsection{Définitions, prévalence et séquence développementale des troubles intériorisés}

Les troubles anxieux regroupent des symptômes tels les attaques de panique, les inquiétudes excessives et les comportements d'évitement. Chez les enfants, on retrouve surtout les phobies simples et l'anxiété de séparation alors que les attaques de panique apparaissent à l'adolescence. Ces troubles touchent entre 12 et $20 \%$ des enfants et des adolescents (Albano, Chorpita et Barlow, 2003). Quant aux troubles dépressifs, ils incluent différents symptômes dont une humeur triste, une perte d'intérêt dans les activités habituelles, une dévalorisation de soi et des problèmes de sommeil et d'appétit. Le trouble dépressif majeur s'observe chez 2 à $3 \%$ des enfants et chez 5 à $9 \%$ des adolescents. Il touche $16 \%$ des adolescents; chez les filles, il peut atteindre jusqu'à $25 \%$ au début du secondaire (Fleming et Offord, 1990). Des études ont montré que les troubles anxieux précédaient l'arrivée des troubles dépressifs dans 72\% des cas (Essau, 2003). Des troubles anxieux sont déjà présents durant l'enfance alors que le trouble dépressif majeur émerge davantage avec la puberté et la transition du primaire au secondaire. C'est lors de cette transition qu'une nette prédominance de dépression apparaît chez les filles comparativement aux garçons.

\subsection{Troubles intériorisés et adaptation à l'école}

Les jeunes qui ont des troubles intériorisés sont souvent ignorés à l'école. D'une part, ils sont peu dérangeants comparativement aux jeunes présentant des troubles extériorisés; d'autre part, les enseignants sont peu habiletés à reconnaître chez leurs élèves les signes de la dépression et de l'anxiété. Or, plusieurs études démontrent les effets négatifs de ces troubles sur le rendement scolaire, notamment parce que leurs symptômes peuvent nuire aux fonctions cognitives telles que la mémoire fonctionnelle, la capacité d'attention et la capacité de résolution de problème (Den Hartog, Derix, Van Bemmel, Kremer et Jolles, 2003). Les élèves présentant des troubles intériorisés sont aussi moins motivés, se sentent plus incompétents et s'absentent davantage que les élèves sans difficulté (Mullins, Chard, Hartman, Bowlby, Rich et Burke, 1995). Les élèves dépressifs rapportent peu de soutien de leurs enseignants (Cheung, 1995) alors que les élèves anxieux et dépressifs rapportent de faibles liens d'affiliation avec leurs pairs dans la classe. De même, les élèves qui rapportent fréquemment de la détresse intériorisée sont identifiés par les enseignants comme ceux qui persévèrent moins face aux difficultés scolaires (Roeser et Eccles, 2000). 


\subsection{Stress associé à la transition du primaire au secondaire et la puberté}

Dans leur théorie de la concordance étape-environnement, Eccles et Midgley (1989; Roeser et Eccles, 2000) décrivent les processus interpersonnels, académiques et organisationnels par lesquels l'école influence le développement de la santé mentale des enfants. Selon ces chercheurs, la transition du primaire au secondaire contribue à l'émergence de certains troubles émotionnels ainsi qu'à la baisse du niveau de motivation des jeunes, car l'école secondaire offre un environnement social qui n'est pas ajusté aux besoins psychologiques de plusieurs adolescents. Des études montrent effectivement que les résultats scolaires chutent lorsque les jeunes passent au secondaire et que la magnitude de cette chute prédit les échecs scolaires subséquents et même le décrochage (Anderman, Maehr et Midgley, 1999). D'autres études confirment que la qualité et la fréquence des interactions entre les adultes et les élèves influencent la présence de symptômes intériorisés chez les élèves (Kuperminc, Leadbeater et Blatt, 2001). Le fait d'être victime d'intimidation serait aussi associé à la dépression et possiblement à la phobie sociale. Entre autres, la dépression jouerait un rôle médiateur entre la victimisation chez les garçons et leur sentiment d'affiliation à leurs pairs en classe (Lepage, Marcotte et Fortin, à paraître). Des études indiquent également que l'école secondaire est davantage axée sur les buts de performance que sur les buts de maîtrise, ces derniers étant associés à un apprentissage plus adapté et à une meilleure adaptation émotionnelle (Midgley, Middleton, Gheen et Kumar, 2002).

Dans une perspective complémentaire, il a été démontré que l'émergence des différences sexuelles dans la dépression est davantage reliée à la maturation pubertaire qu'à l'âge chronologique et s'explique, notamment, par une image corporelle et une estime de soi plus faibles chez les filles causées par une appréciation plus négative des changements pubertaires (Wichstrom, 1999). Nolen-Hoeksema et Girgus (1994) proposent que la synchronie entre l'arrivée de la puberté, qui se produit deux ans plus tôt chez les filles que chez les garçons, et la transition du primaire au secondaire constitue un facteur stressant associé à l'émergence de taux élevés de dépression chez les filles. En accord avec ce modèle, Ge, Conger et Elder (1996) ont observé que les filles qui sont pubères de façon précoce, c'est-à-dire à 11,9 ans en moyenne, donc en synchronie avec la transition du primaire au secondaire, sont celles qui montrent le plus haut niveau de détresse psychologique en $7^{\mathrm{e}}$ année ainsi que systématiquement en $8^{\mathrm{e}}, 9^{\mathrm{e}}$ et $10^{\mathrm{e}}$ année. Nos travaux confirment que l'image corporelle, l'estime de soi et les événements de vie stressants agissent comme des variables médiatrices dans la relation entre le sexe et la dépression, de même qu'entre la maturation pubertaire et la dépression chez les élèves vivant la transition du primaire au secondaire (Marcotte, Fortin, Potvin et Papillon, 2002). Les adolescentes québécoises vivent leurs premières menstruations en moyenne à 12,7 ans, ce qui est comparable aux moyennes américaines. Cependant, nous ne savons pas encore quels sont les facteurs scolaires spécifiques reliés à la transition du primaire au secondaire qui agissent comme des éléments stressants en interaction avec la puberté.

\subsection{Facteurs personnels et familiaux}

Au plan personnel, les jeunes dépressifs et les jeunes anxieux rapportent plus d'isolement social et moins de soutien d'amis que les jeunes sans difficulté (Kaltiala-Heino, Rimpela, Rantanen et Laippala, 2001). Un manque d'habiletés sociales et d'habiletés de résolution de problèmes interpersonnels chez les jeunes dépressifs a été suggéré par certains auteurs (Cole, 
1990) : la présence d'un épisode dépressif pendant l'enfance ou l'adolescence viendrait hypothéquer l'acquisition de telles habiletés, cette lacune devenant à son tour un facteur de risque pour des épisodes subséquents. Les jeunes dépressifs sont très préoccupés par la crainte du rejet et par leur définition de soi, ce qui s'exprime à travers une critique de soi sévère et un faible sentiment d'efficacité (Kuperminc et al., 2001). Les jeunes anxieux sont plutôt préoccupés par des craintes, des peurs face aux nouvelles situations et utilisent des comportements d'évitement. Tous ces jeunes tendent à utiliser des distorsions cognitives face à la perception d'eux-mêmes, du monde et de l'avenir et ont des attitudes dysfonctionnelles face à la réussite.

Les facteurs familiaux sont fortement associés à la présence de troubles intériorisés chez les enfants et les adolescents. La prévalence de ces troubles est environ quatre fois plus élevée chez les enfants de parents dépressifs en comparaison des enfants de parents non dépressifs (Hammen, 1997). Le milieu familial des jeunes dépressifs se caractérise par des pratiques parentales peu adéquates, la mésentente familiale et conjugale, le divorce et l'abus. Le manque de cohésion familiale est souligné par plusieurs auteurs (Cole et McPherson, 1993). Chez les enfants anxieux, les écrits font plutôt état de pratiques parentales intrusives et contrôlantes, ainsi que d'une relation parent-enfant teintée de surprotection et de rejet (Dadds et Roth, 2001). La présence de troubles anxieux chez les parents est également largement documentée. Les réactions anxieuses des parents face aux nouvelles situations comme l'entrée à l'école, font en sorte de renforcer les comportements d'évitement et le développement de symptômes anxieux chez l'enfant. Cependant, à ce jour, nous connaissons peu les processus par lesquels le vécu familial des enfants et des adolescents ayant des troubles intériorisés affecte leur rendement scolaire, ni les facteurs scolaires qui peuvent réduire les effets négatifs de la dysfonction familiale. Les jeunes dont les parents se sont séparés pendant la transition vers le secondaire seraient particulièrement à risque d'échec scolaire et de dépression (Zubernis, Wright-Cassidy, Gillham, Reivich et Jaycox, 1999). L'objectif de cette étude est de comparer les facteurs personnels, scolaires et familiaux associés aux troubles intériorisés à la fin du primaire et au début du secondaire.

\section{Méthode}

\subsection{Participants et procédure}

Quatre cent quatre vingt dix-neuf élèves de $6^{\mathrm{e}}$ année (235 filles et 264 garçons; âge moyen: $11,2$ ans; é.t. $=0,5)$ provenant de 12 écoles primaires ont participé à la première étude. Quatre cent soixante dix-huit élèves de $2^{\mathrm{e}}$ secondaire ( 270 filles et 208 garçons; âge moyen: 13,4 ; é.t. $=$ 0,6 ) provenant de quatre écoles secondaires ont participé à la deuxième étude. Les participants proviennent de la Mauricie et de la rive sud de Montréal. Les taux de participation ont été respectivement de $80 \%$ et de $94 \%$ pour la première et la deuxième étude.

La participation des élèves était volontaire et requérait le consentement écrit des parents ou tuteurs. Les questionnaires ont été complétés dans le cadre de cours réguliers durant des périodes d'environ 60 minutes. Les enseignants ont aussi été invités à compléter le Système d'évaluation des comportements pour enfants pour chacun des participants. 


\subsection{Instruments de mesure}

\subsubsection{Troubles intériorisés}

Les symptômes dépressifs ont été identifiés avec la version française du Beck Depression Inventory (Beck, 1978) pour les participants de $2^{\mathrm{e}}$ secondaire et de la version révisée (BDI-II) (Beck, Steer et Brown, 1996) pour ceux de $6^{\mathrm{e}}$ année du primaire. Les symptômes anxieux ont été identifiés avec le Dominique Adolescent Interactif en $6^{\mathrm{e}}$ année du primaire et le Beck Anxiety Scale (Beck, Epstein, Brown et Steer, 1988) en $2^{\mathrm{e}}$ secondaire.

\subsubsection{Variables personnelles}

L'image corporelle a été évaluée à l'aide de la sous-échelle du Self-Perception Profile for Adolescents (Harter, 1988) en $6^{\mathrm{e}}$ année du primaire et du Offer Self-Image Questionnaire (Offer, Ostrov et Howard, 1981) en 2 ${ }^{\mathrm{e}}$ secondaire. Le Dysfunctionnal Attitudes Scale (Weissman et Beck, 1978) a servi à l'évaluation des distorsions cognitives liées à la dépendance, la réussite et l'autocontrôle. Le Perceived Social Support-Friends (Procidano et Heller, 1983) a mesuré le soutien des amis. La manifestation des délits a été évaluée avec le Questionnaire de la délinquance autorévélée (Leblanc, 1994).

\subsubsection{Variables familiales}

Le Family Environment Scale (Moos et Moos, 1981) a permis d'identifier les caractéristiques familiales comme la cohésion, l'expression, les conflits, l'organisation et le contrôle des sphères familiales.

\subsubsection{Variables scolaires}

Le Classroom Environment Scale (Moos et Trickett, 1987) a été utilisé auprès des élèves pour évaluer différentes caractéristiques du climat de classe dont l'engagement, l'affiliation, le soutien de l'enseignant, l'orientation vers la tâche, la compétition, l'ordre et l'organisation, la clarté des règlements, le contrôle de l'enseignant et l'innovation. Les enseignants ont évalué les difficultés d'adaptation et les problèmes de comportement des élèves avec la version de l'enseignant du Behavior Assessment System for Children (Reynolds et Kamphaus, 1992, 1998). Enfin, les résultats en français et en mathématiques ainsi que le taux d'absentéisme ont été recueillis à partir des dossiers des participants.

\section{Résultats}

Pour chaque échantillon, des comparaisons ont d'abord été effectuées entre les élèves dépressifs/non dépressifs (tableau 1), puis entre les élèves anxieux/non anxieux (tableau 2), à l'aide d'analyses de variance. Les sous-groupes ont été formés à partir des points de coupure suggérés pour les instruments utilisés pour chacune des variables indépendantes. 


\section{Tableau 1}

Caractéristiques qui distinguent les élèves dépressifs et non dépressifs à la fin du primaire et au début du secondaire

\begin{tabular}{|c|c|c|c|c|c|c|}
\hline \multirow[t]{3}{*}{ Caractéristiques } & \multicolumn{3}{|c|}{ Élèves $6^{\mathrm{e}}$ année du primaire } & \multicolumn{3}{|c|}{ Élèves $2^{\mathrm{e}}$ secondaire } \\
\hline & Dépressifs & $\begin{array}{c}\text { Non } \\
\text { dépressifs }\end{array}$ & $\mathrm{F}$ & Dépressifs & \begin{tabular}{|c|} 
Non \\
dépressifs
\end{tabular} & $\mathrm{F}$ \\
\hline & M (é.t.) & M (é.t.) & & M (é.t.) & M (é.t.) & \\
\hline Image corporelle négative & $12,2(4,2)$ & $15,5(3,6)$ & $14,7 * * *$ & $3,6(0,7)$ & $3,5(0,5)$ & $1,0^{*}$ \\
\hline Distorsions cognitives reliées à la réussite & $30,9(10,5)$ & $25,5(9,6)$ & $6,8^{* *}$ & $29,4(8,2)$ & $25,7(7,8)$ & $31,1 * * *$ \\
\hline Distorsions cognitives reliées à la dépendance & $33,3(10,4)$ & $26,5(7,9)$ & $15,4^{* * *}$ & $33,8(8,7)$ & $28,9(6,8)$ & $20,9^{* * *}$ \\
\hline Soutien des amis & & & & $90,7(15,5)$ & $94,3(15,5)$ & $11,3^{* * *}$ \\
\hline Fréquence élevée des délits & $5,6(4,3)$ & $2,2(3,0)$ & $20,6^{* * *}$ & $6,1(7,5)$ & $3,34(3,8)$ & $27,9^{* * *}$ \\
\hline Cohésion familiale & $39,2(17,3)$ & $55,3(13,0)$ & $25,9^{* * *}$ & $4,2(2,6)$ & $7,2(1,7)$ & $137,1 * * *$ \\
\hline Conflits familiaux & $58,2(13,5)$ & $44,5(11,6)$ & $24,9 * * *$ & $5,4(2,3)$ & $2,5(2,3)$ & $96,8 * * *$ \\
\hline Organisation familiale & $46,7(9,7)$ & $53,1(8,0)$ & $7,4^{* *}$ & $4,8(1,9)$ & $6,2(1,7)$ & $49,1^{* *}$ \\
\hline Expression des sentiments en famille & & & & $4,6(1,7)$ & $5,9(1,5)$ & $55,5^{* * *}$ \\
\hline Contrôle parental & & & & $4,9(2,1)$ & $3,8(1,8)$ & $20,8^{* * *}$ \\
\hline $\begin{array}{l}\text { Auto-évaluation négative du rendement } \\
\text { scolaire }\end{array}$ & & & & $1,9(0,6)$ & $1,8(0,6)$ & $19,6^{* * *}$ \\
\hline Ordre et organisation dans la classe & $1,7(1,1)$ & $2,3(1,2)$ & $4,7^{*}$ & & & \\
\hline Clarté des règlements dans la classe & $3,0(1,2)$ & $3,5(0,8)$ & $6,6^{*}$ & & & \\
\hline Affiliation dans la classe & & & & $3,1(1,0)$ & $2,8(1,3)$ & $7,3 * *$ \\
\hline $\begin{array}{l}\text { Difficultés d'apprentissage évaluées par } \\
\text { l'enseignant }\end{array}$ & $8,0(5,6)$ & $5,3(4,9)$ & $6,7^{* *}$ & $6,6(4,8)$ & $5,6(5,7)$ & $9,3 * * *$ \\
\hline $\begin{array}{l}\text { Problèmes d'attention évalués par } \\
\text { l'enseignant }\end{array}$ & $8,0(4,2)$ & $5,1(4,3)$ & $11,7^{* * *}$ & $6,9(4,2)$ & $5,7(5,0)$ & $10,6^{* * *}$ \\
\hline $\begin{array}{l}\text { Faibles habiletés sociales évaluées par } \\
\text { l'enseignant }\end{array}$ & $13,2(6,9)$ & $16,7(7,8)$ & $5,0^{*}$ & $15,2(7,1)$ & $17,5(7,4)$ & $13,5^{* * *}$ \\
\hline Leadership évalué par l'enseignant & $9,2(4,6)$ & $11,7(5,3)$ & $4,8^{*}$ & $9,2(5,2)$ & $11,5(5,6)$ & $16,0^{* * *}$ \\
\hline $\begin{array}{l}\text { Faibles habiletés d'étude évaluées par } \\
\text { l'enseignant }\end{array}$ & $15,6(7,5)$ & $21,1(8,0)$ & $12,1 * * *$ & $16,6(7,5)$ & $20,6(9,1)$ & $21,2^{* * *}$ \\
\hline $\begin{array}{l}\text { Troubles du comportement évalués par } \\
\text { l'enseignant }\end{array}$ & $2,5(3,3)$ & $1,4(2,0)$ & $5,9^{*}$ & $4,3(4,6)$ & $2,3(3,5)$ & $23,7^{* * *}$ \\
\hline Rendement scolaire en français & $2,6(0,7)$ & $3,1(0,7)$ & $9,7 * *$ & $69,0(10,6)$ & $73,7(11,1)$ & $23,2^{* * *}$ \\
\hline Rendement scolaire en mathématiques & & & & $58,2(16,5)$ & $69,4(14,8)$ & $29,1 * * *$ \\
\hline Absentéisme & & & & $37,3(46,0)$ & $23,0(29,2)$ & $10,6^{* * *}$ \\
\hline
\end{tabular}

Légende: ${ }^{*} \mathrm{p}<0,05 ; * * \mathrm{p}<0,01 ; * * * \mathrm{p}<0,001$.

\subsection{Comparaison des élèves dépressifs/non dépressifs}

Au plan des facteurs personnels, les analyses révèlent que les distorsions cognitives reliées à la réussite («Si je ne réussis pas totalement, c'est comme si j’avais échoué.») et à la dépendance («Mon bonheur dépend plus des autres que de moi.») sont présentes chez les élèves dépressifs autant à la fin du primaire qu'au début du secondaire. On constate également la présence d'une image corporelle plus négative chez les dépressifs. Ces derniers commettent davantage de délits que les jeunes non dépressifs. Un soutien plus faible de la part des amis est rapporté chez les jeunes dépressifs de l'échantillon du début du secondaire, mais non chez les jeunes de la fin du primaire. 
Tableau 2

Caractéristiques qui distinguent les élèves anxieux et non anxieux à la fin du primaire et au début du secondaire

\begin{tabular}{|c|c|c|c|c|c|c|}
\hline \multirow[t]{3}{*}{ Caractéristiques } & \multicolumn{3}{|c|}{ Élèves $6^{\mathrm{e}}$ année du primaire } & \multicolumn{3}{|c|}{ Élèves $2^{\mathrm{e}}$ secondaire } \\
\hline & Anxieux & \begin{tabular}{|c|} 
Non \\
anxieux
\end{tabular} & $\mathrm{F}$ & Anxieux & \begin{tabular}{c|} 
Non \\
anxieux
\end{tabular} & $\mathrm{F}$ \\
\hline & M (é.t.) & M (é.t.) & & M (é.t.) & M (é.t.) & \\
\hline Image corporelle négative & $10,8(3,8)$ & $15,3(3,9)$ & $22,3 * * *$ & & & \\
\hline $\begin{array}{l}\text { Distorsions cognitives reliées } \\
\text { à la réussite }\end{array}$ & $30,7(13,2)$ & $27,8(10,0)$ & $5,3^{*}$ & $28,9(8,0)$ & $36,1(8,0)$ & $8,4 * * *$ \\
\hline $\begin{array}{l}\text { Distorsions cognitives reliées } \\
\text { à la dépendance }\end{array}$ & $32,3(8,1)$ & $27,9(8,0)$ & $7,7^{* *}$ & $32,8(7,9)$ & $29,3(7,6)$ & $8,1 * * *$ \\
\hline Soutien des amis & $83,6(16,6)$ & $92,3(15,4)$ & $11,8^{* * *}$ & & & \\
\hline Fréquence élevée des délits & & & & $5,9(7,1)$ & $3,6(4,3)$ & $11,9^{* * *}$ \\
\hline Cohésion familiale & $38,2(15,9)$ & $51,1(15,8)$ & $10,3^{* *}$ & $5,5(2,6)$ & $7,0(2,1)$ & $20,3^{* * *}$ \\
\hline Conflits familiaux & $62,7(12,4)$ & $48,7(13,3)$ & $19,1^{* * *}$ & $4,6(2,6)$ & $2,8(2,5)$ & $21,7 * * *$ \\
\hline Organisation familiale & & & & $5,4(2,0)$ & $6,0(1,7)$ & $4,9 *$ \\
\hline Contrôle familial & $55,2(10,2)$ & $49,0(11,0)$ & $6,0^{*}$ & $4,6(2,1)$ & $3,9(2,0)$ & $4,5^{*}$ \\
\hline Expression des sentiments en famille & & & & $5,0(1,8)$ & $5,8(1,6)$ & $10,1 * * *$ \\
\hline Compétition dans la classe & $1,7(0,9)$ & $1,6(0,9)$ & $4,9^{*}$ & $1,5(1,0)$ & $1,2(0,8)$ & $11,6^{* * *}$ \\
\hline Ordre et organisation dans la classe & $1,8(1,0)$ & $2,2(1,1)$ & $5,4^{*}$ & $1,1(1,0)$ & $1,5(1,3)$ & $4,7^{*}$ \\
\hline Affiliation dans la classe & $2,8(1,2)$ & $3,3(0,9)$ & $5,3^{*}$ & & & \\
\hline Engagement dans la classe & $2,4(0,9)$ & $2,9(1,0)$ & $6,4^{*}$ & $1,1(1,1)$ & $1,5(1,2)$ & $7,4^{* *}$ \\
\hline $\begin{array}{l}\text { Difficultés d'apprentissage évaluées par } \\
\text { l'enseignant }\end{array}$ & & & & $6,5(5,1)$ & $5,2(5,3)$ & $4,2^{*}$ \\
\hline $\begin{array}{l}\text { Troubles du comportement évalués par } \\
\text { l'enseignant }\end{array}$ & & & & $3,5(4,1)$ & $2,4(3,3)$ & $5,4^{*}$ \\
\hline Rendement scolaire en français & & & & $70,5(11,8)$ & $73,1(10,8)$ & $3,9^{*}$ \\
\hline Absentéisme & & & & $33,1(49,1)$ & $21,3(25,3)$ & $6,2 * *$ \\
\hline
\end{tabular}

Légende: ${ }^{*} \mathrm{p}<0,05 ; * * \mathrm{p}<0,01 ; * * * \mathrm{p}<0,001$.

Au plan familial, les élèves dépressifs de la fin du primaire rapportent une plus faible cohésion de la famille, davantage de conflits familiaux et une organisation familiale plus faible que les jeunes non dépressifs. Chez les élèves dépressifs du début du secondaire, s'ajoutent les dimensions reliées à l'expression des sentiments et au contrôle parental.

Au plan scolaire, les mesures autorévélées qui distinguent les jeunes dépressifs diffèrent selon les échantillons. En $6^{\mathrm{e}}$ année, les élèves dépressifs perçoivent moins d'ordre, d'organisation et de clarté des règlements dans la classe, alors qu'au début du secondaire, ils perçoivent une plus faible affiliation aux pairs et un plus faible rendement scolaire. Les élèves dépressifs des deux échantillons se distinguent toutefois de ceux non dépressifs sur l'ensemble des dimensions évaluées par les enseignants. Enfin, le rendement scolaire en français est plus faible chez les élèves dépressifs de chaque échantillon, alors que celui en mathématiques est plus faible au début du secondaire seulement. Dans ce dernier groupe, le taux d'absentéisme est aussi plus élevé chez les dépressifs. 


\subsection{Comparaison des élèves anxieux/non anxieux}

Pour les facteurs personnels, les analyses révèlent que les distorsions cognitives reliées à la réussite et à la dépendance sont présentes chez les élèves anxieux autant à la fin du primaire qu'au début du secondaire. Une image corporelle plus négative ainsi qu'un soutien plus faible des amis sont rapportés en $6^{\mathrm{e}}$ année seulement, alors qu'une fréquence plus élevée de délits chez les élèves anxieux est rapportée au début du secondaire.

Au plan familial, les jeunes anxieux et non anxieux des deux échantillons se distinguent sur des dimensions reliées à la cohésion familiale, aux conflits et au contrôle parental. Les élèves anxieux du début du secondaire se distinguent aussi sur les dimensions reliées à l'organisation familiale et à l'expression des sentiments.

Au plan scolaire, plusieurs mesures autorévélées caractérisent les jeunes anxieux des deux échantillons, dont la présence de compétition dans la classe, le manque d'ordre et d'organisation et un faible engagement. L'affiliation aux pairs distinguent les élèves anxieux à la fin du primaire seulement. Parmi les dimensions évaluées par l'enseignant, seule la présence de difficultés d'apprentissage et de troubles comportementaux distinguent les élèves anxieux, et ce, au début du secondaire seulement. Ces élèves ont un rendement scolaire plus faible en français et s'absentent davantage de l'école.

\section{Discussion}

Les résultats suggèrent que plusieurs facteurs personnels, familiaux et scolaires distinguent les élèves dépressifs des non dépressifs ainsi que les élèves anxieux des non anxieux tant à la fin du primaire qu'au début du secondaire. D'abord, les jeunes dépressifs tout comme les jeunes anxieux utilisent des distorsions cognitives dans leur interprétation des situations, ce qui peut les rendre plus vulnérables à certains stress en milieu scolaire. Ces jeunes tendent à adopter une attitude de dépendance et à avoir une attitude irréaliste face à la réussite. Par exemple, une note moyenne pourra être interprétée comme un échec complet, alors que la perte d'une relation privilégiée avec l'enseignant lors du passage au secondaire pourra être interprétée comme du rejet. L'approche cognitive devient ainsi un modèle d'intervention pertinent qui s'est d'ailleurs avéré efficace auprès des jeunes de milieu scolaire (Marcotte, 2000; Platts et Williamson, 2000).

Le soutien des amis semble associé différemment aux élèves dépressifs et aux élèves anxieux. Ce soutien distingue les jeunes anxieux des jeunes non anxieux à la fin du primaire et est accompagné d'un sentiment de manque d'affiliation avec les pairs dans la classe. Chez les jeunes dépressifs, c'est plutôt au début du secondaire que cette différence s'observe. Elle s'accompagne aussi d'un sentiment de manque d'affiliation, c'est-à-dire de liens d'amitié plus faibles avec les autres élèves de la classe. L'évaluation des enseignants corrobore cet isolement social des jeunes dépressifs. Ces derniers évaluent les jeunes dépressifs comme présentant de faibles habiletés sociales et moins d'habiletés de leadership. Ce manque d'habiletés chez les jeunes dépressifs est également observé par les enseignants dès la fin du primaire, ce qui suggère une piste d'intervention à considérer. 
Nos résultats confirment l'influence du contexte familial sur la présence de troubles intériorisés et indiquent qu'une faible cohésion familiale et la présence de conflits sont associées autant aux troubles anxieux que dépressifs, et ce, tant avant la transition vers le secondaire qu'après cette transition. De plus, il semble que les adolescents dépressifs et anxieux éprouvent le besoin d'exprimer davantage leurs sentiments dans leur famille une fois l'adolescence bien installée. On peut penser que, la maturation cognitive et émotionnelle prenant place avec l'adolescence, les garçons et les filles ressentent le besoin d'exprimer leurs besoins émotionnels davantage par le moyen d'une expression verbale plus élaborée en comparaison avec les enfants du primaire.

Le rendement scolaire des jeunes dépressifs est affecté plus particulièrement au début du secondaire alors que la dépression est aussi associée à une perception négative du rendement. Pour les élèves anxieux, seul le rendement en français au début du secondaire est plus faible en comparaison aux élèves non anxieux. Les dimensions auto-évaluées par les élèves diffèrent selon les échantillons et selon l'ordre scolaire. Un sentiment de compétition entre les élèves ainsi qu'un faible engagement sont associés aux troubles anxieux, mais non aux troubles dépressifs. Les élèves anxieux témoignent davantage d'une perception qu'il leur est difficile d'obtenir des bonnes notes et que l'accent dans la classe est mis sur la course aux meilleurs notes possible. Ils rapportent également un degré moindre d'intérêt et de participation, c'est-à-dire qu'ils se sentent moins engagés face aux activités se déroulant en classe. On constate également que plusieurs difficultés de fonctionnement en classe sont associées par les enseignants aux élèves dépressifs, alors que seulement deux dimensions, soit les difficultés d'apprentissage et les troubles du comportement, sont associées aux symptômes anxieux au début du secondaire seulement.

\section{Conclusion et limites}

Les résultats de ces deux études démontrent donc que les symptômes de troubles intériorisés sont associés à des difficultés d'adaptation au plan personnel, social, familial et scolaire et mettent en lumière l'importance d'examiner de façon plus approfondie la relation entre la santé mentale des jeunes et leur réussite scolaire et éducative. Les jeunes dépressifs et anxieux obtiennent un rendement plus faible et rapportent se sentir moins adaptés au milieu scolaire, en plus de vivre des difficultés au sein de leur famille. Les difficultés de ces jeunes semblent partiellement observées par les enseignants. Cependant, étant donné que ces jeunes, plus particulièrement les élèves dépressifs, rapportent plus de délits que les jeunes sans difficulté et que la présence de troubles extériorisés n'a pas été contrôlée dans ces études, il est difficile d'identifier si les observations des enseignants sont associées directement aux troubles intériorisés. L'identification des dimensions du fonctionnement scolaire spécifiques aux troubles intériorisés demeure nécessaire afin d'élaborer des modèles d'intervention propres à ces problématiques.

Parmi les limites, il faut aussi mentionner la diversité des mesures utilisées, notamment dans l'identification des symptômes de dépression et d'anxiété, qui n'ont pu être identiques dans les échantillons. Également, la spécificité des troubles anxieux n'a pu être considérée dans les analyses ni les différences sexuelles. Une analyse des caractéristiques associées aux troubles intériorisés à partir d'un devis longitudinal couvrant la transition du primaire au secondaire sera nécessaire dans les futures recherches. 


\section{Références}

Albano, A.M., Chorpita, B.F. et Barlow, D.H. (2003). Childhood anxiety disorders. In E.J. Mash et R.A. Barkley (dir.), Child psychopathology (2e édition) (p. 279-329). New York, NY : Guilford Press.

Anderman, E.M., Maehr, M.L. et Midgley, C. (1999). Declining motivation after the transition to middle school: Schools can make a difference. Journal of Research and Development in Education, 32(3), 131-147.

Atkinson, M. et Hornby, G. (2002). Mental health handbook for schools. New Fetter Lane, NY: Routledge Falmer.

Beck, A.T. (1978). Depression Inventory. Philadelphia: Center for Cognitive Therapy.

Beck, A.T., Epstein, N., Brown, G. K et Steer, R.A. (1988). An inventory for measuring clinical anxiety: Psychometric properties. Journal of Consulting and Clinical Psychology, 56(6), 893-897.

Beck, A.T., Steer, R.A. et Brown, G.K. (1996). The Beck Depression Inventory Manual (2e édition). San Antonio, TX: The Psychological Corporation.

Cheung, S.K. (1995). Life events, classroom environment, achievement expectation and depression among early adolescents. Social Behavior and Personnality, 23(1), 83-92.

Cole, D.A. (1990). Relation of social and academic competence to depressive symptoms in childhood. Journal of Abnormal Psychology, 99(4), 422-429.

Cole, D.A. et McPherson, A.E. (1993). Relation of family subtypes to adolescent depression: Implementing a new family assessment strategy. Journal of Family Psychology, 7(1), 119-133.

Dadds, M.R. et Roth, J.H. (2001). Family processes in the development of anxiety problems. In M.W. Vasey et M.R. Dadds (dir.), The developmental psychopathology of anxiety (p. 278-303). New York, NY : Oxford University Press.

Den Hartog, H.M., Derix, M.M., Van Bemmel, A.L., Kremer, B. et Jolles, J. (2003). Cognitive functioning in young and middle-aged unmedicated out-patients with major depression: Testing the effort and cognitive speed hypotheses. Psychological Medecine, 33(3), 1443-1451.

Eccles, J.S. et Midgley, C. (1989). Stage/environment fit: Developmentally appropriate classrooms for early adolescents. In R.E. Ames et C. Ames (dir.), Research on motivation in education (Vol. 3, p. 139-186). New York, NY: Academic Press.

Essau, C.A. (2003). Comorbidity of anxiety disorders in adolescents. Depression and Anxiety, 18(1), 1-6.

Fleming, J.E. et Offord, D.R. (1990). Epidemiology of childhood depressive disorders : A critical review. Journal of the American Academy of Child and Adolescent Psychiatry, 29(4), 571-580.

Ge, X., Conger, R.D. et Elder, G.H. (1996). Coming of age too early: Pubertal influences on girl's vulnerability to psychological distress. Child Development, 67(6), 3386-3400.

Hammen, C.L. (1997). Depression. Hove: Psychology Press.

Harter, S. (1988). Manual for the self-perception profile for adolescents. Denver, CO: University of Denver Press.

Kaltiala-Heino, R., Rimpela, M., Rantanen, P. et Laippala, P. (2001). Adolescent depression: The role of discontinuities in life course and social support. Journal of Affective Disorders, 64(2-3), 155-166.

Kuperminc, G.P., Leadbeater, B.J. et Blatt, S.J. (2001). School social climate and individual differences in vulnerability to psychopathology among middle school students. Journal of School Psychology, 39(2), 141-159.

Leblanc, M. (1994). MASPAQ, Manuel sur des mesures de l'adaptation sociale et personnelle pour les adolescents québécois. Communication présentée au GRIP, École de psychoéducation, Université de Montréal, avril.

Lepage, C., Marcotte, D., Fortin, L., Royer, É. et Potvin, P. (à paraître). Analyse d'un modèle médiateur de l'intimidation chez des adolescents de $5^{\mathrm{e}}$ secondaire. Scientia Paedagogica Experimentalis.

Marcotte, D. (2000). La prévention de la dépression chez les enfants et adolescents. In F. Vitaro et C. Gagnon (dir.), Prévention des problèmes d'adaptation chez les jeunes (Tome 1, p. 221-270). Québec: Presse de 1'Université du Québec.

Marcotte, D., Fortin, L., Potvin, P. et Papillon, M. (2002). The mediating role of gender- typed characteristics, pubertal status, self-esteem, body image and stressful life events in the emergence of gender differences in depression during adolescence. Journal of Emotional and Behavioral Disorders, 10(1), 29-42. 
Midgley, C., Middleton, M.J., Gheen, M.H. et Kumar, R. (2002). Stage-environment fit revisited: A goal theory approach to examining school transitions. In C. Midgley (dir.), Goals, goal structures and patterns of adaptive learning (p. 109-142). Mahwah, NJ: Lawrence Erlbaum.

Moos, R.H. et Moos, B.A. (1981). Manual for the Family Environment Scale. Palo Alto, CA: Consulting Psychologists Press.

Moos, R.H. et Tricket, E.J. (1987). Classroom Environment Scale Manual (2édition). Palo Alto, CA : Consulting Psychologists Press.

Mullins, L.L., Chard, S.R., Hartman, V.L., Bowlby, D., Rich, L. et Burke, C. (1995). The relationship between depressive symptomatology in school children and the social responses of teachers. Journal of Clinical Child Psychology, 24(4), 474-482.

Nolen-Hoeksema, S. et Girgus, J. (1994). The emergence of gender differences in depression during adolescence. Psychological Bulletin, 115(3), 424-443.

Offer, D., Ostrov, E. et Howard, K.I., (1981). The adolescent : A psychological self-portrait. New York, NY : Basic Books.

Platts, J. et Williamson, Y. (2000). The use of cognitive-behavioural therapy for counseling in schools. In N. Barwick (dir.), Clinical counseling in schools (p. 96- 107). Philadelphia, PA: Taylor and Francis.

Procidano, M.E. et Heller, K. (1983) Measures of perceived social support from friends and from family: Three validation studies. American Journal of Community Psychology, 11(1), 1-24.

Reynolds, C.R. et Kamphaus, R.W. (1998). Behavior Assessment System for Children. Circle Pines, MN : American Guidance Service.

Roeser, R.W. et Eccles, J.S. (2000). Schooling and mental health. In A.J. Sameroff, M. Lewis et S.M. Miller (dir.), Handbook of developmental psychopathology (2 $2^{\mathrm{e}}$ édition) (p. 135-156). New York, NY: Academic/Plenum Publishers.

Weissman, A. et Beck, A.T. (1978). Development and validation of the Dysfunctional Attitude Scale. Communication presentée à l'Annual meeting of theAssociation for the Advancement of Behaviour Therapy, Chicago, avril.

Wichstrom, L. (1999). The emergence of gender difference in depressed mood during adolescence: The role of intensified gender socialization. Developmental Psychology, 35(1), 232-245.

Zubernis, L.S., Wright-Cassidy, K., Gillham, J.E., Reivich, K.J. et Jaycox, L.H. (1999). Prevention of depressive symptoms in preadolescent children of divorce. Journal of Divorce and Remarriage, 30(1-2), 11-36. 